\title{
Clustering-BASEd ROUTING FOR WIRELESS SENSOR NETWORKS IN SMART GRID ENVIRONMENT
}

\author{
Laila Nassef $^{1,2}$, Reemah El-Habshi ${ }^{1}$ and Linta Jose ${ }^{1}$ \\ ${ }^{1}$ Department of Computer Science, Faculty of Computing and Information Technology, \\ King Abdulaziz University, Jeddah, Saudi Arabia \\ ${ }^{2}$ Department of Computer Sciences and Information, Institute of Statistical Studies and \\ Research, Cairo University, Egypt
}

\begin{abstract}
Wireless Sensor Networks (WSN) is widely deployed in different fields of applications of smart grid to provide reliable monitoring and controlling of the electric power grid. The objective of this paper is simulate and analyze impact of various smart grid environments on performance of four different WSN routing protocols namely the Low Energy Adaptive Clustering Hierarchy (LEACH) and Centralized LEACH (LEACT-C) as well as other two conventional protocols namely Minimum Transmission Energy (MTE) and Static Clustering. This analysis would be beneficial in making the correct choice of WSN routing protocols for various smart grid applications. The performance of the four protocols is simulated using NS-2 network simulation on Ubuntu. The results are analyzed and compared using number of data signals received at base station, energy consumption, and network lifetime as performance metrics. The results show that the performance of various protocols in the smart grid environments have deteriorated due log normal channel characteristics and consequently network lifetime have decreased significantly. The results also indicate that clustering based routing protocols have more advantageous over conventional protocols; MTE and static clustering. Also, centralized clustering approach is more effective as it distributes energy dissipation evenly throughout the sensor nodes which reduce energy consumption and prolong the networks' lifetime. This approach is more effective in delivering data to base station because it has global knowledge of the location and energy of all the nodes in the network.
\end{abstract}

\section{KEYWORDS}

Wireless Sensor Networks; Clustering; Energy Consumption, Network Lifetime.

\section{INTRODUCTION}

The current power grid suffers from lack of effective communications, monitoring, fault diagnostics, and automation, which increases the possibility of region wide system breakdown. Smart grid [1] is a new generation of electric power network that modernizes electric power grid network using advanced sensors, and distributed computing technologies to improve the efficiency, reliability and safety of power delivery. Electric power grid consists of three main subsystems: power generation, power transmission and distribution, and customer facilities. Smart grid needs online monitoring, diagnostics and protection to ensure better control by incorporating automation and self-healing capabilities. Recently, Wireless Sensor Networks (WSNs) [1] have been recognized as a promising technology to achieve seamless, energy efficient, reliable and low cost monitoring and control of the smart grid. WSNs are applied through the three main subsystems of the smart grid. Though WSNs brings about many advantages to the smart grid technology, it also brings up many challenges because of unique characteristics, resource constraints, and the harsh and complex electric-power environment. 
Therefore this paper investigate performance of various cluster based routing protocols in different smart grid environments.

Sensors are small, inexpensive, self powered devices that can sense and communicate with each other for the purpose of collecting local information to make global decisions about their physical environment [2]. Information is aggregated to a local processing and control system, which in turn can communicate with a remote system using any of the various external networks available. In WSNs, nodes can send their data to the Base Station (BS) using routing protocols that are broken down into three categories. First, direct communication (DC) [3], which is the simplest protocol, where sensor nodes send data directly to the BS. In direct communication protocol, sensor nodes, which are far from the BS, dissipate faster than others do because they send their data to the BS directly. The second category involve Minimum Transmission Energy (MTE) protocols [4], where nodes route data to the BS through intermediate nodes, each node acting as a router for the other nodes [5].

The limited energy supplies of WSN nodes, unreliable communication due to the wireless medium, and the need for self-configuration impose constraints on network's lifetime. These constraints along with limited computational and memory storage represent a challenge to keep network's protocols as light weight as possible [6]. These energy constraints necessitate the need for energy efficiency routing protocols and various energy efficient protocols have been proposed to solve problems in conventional routing protocols. One of the energy efficient techniques to extend the lifetime of WSNs is clustering [7]. Therefore, the third category are made up of clustering protocols, where sensor nodes are organized into independent clusters, each with a Cluster Head $(\mathrm{CH})$ node and a number of member nodes to collect data from their environment and forward it to the $\mathrm{CH}$. $\mathrm{CH}$ collect and aggregate information from sensors in their own cluster and forward information to the BS, which collects and processes data in order to act either as a supervisory control station or as an access point for a human interface or to act as a gateway to be connected to remote stations. For efficiently maintain, the routing path between the BS and sensor nodes, various types of clustering protocols can be used. Nodes in static clustering are organized into clusters that communicate with a local BS that transmit the data to the global BS, where it is accessed by the end-user [8].

The Low Energy Adaptive Clustering Hierarchy (LEACH) [9] is a cluster based hierarchical algorithm. It is a cross layer protocol architecture that integrates energy efficient cluster based routing and media access together with application specific data aggregation to achieve good performance [10]. LEACH forms clusters by using a distributed algorithm, where nodes make autonomous decisions without any centralized intervention. In $\mathrm{LEACH}$, distributed cluster formation can be done without knowing the exact location of any of the nodes in the network and any sensor node can act as $\mathrm{CH}$. The selection of $\mathrm{CH}$ rotate among nodes to distribute energy evenly in the whole network. Once a node declares itself the $\mathrm{CH}$, the nearby nodes join he cluster and send their data to $\mathrm{CH}$ in their assigned time slot.

Unlike the LEACH, LEACH-C [11] utilizes a central BS for the formation of CHs. During set-up phase of LEACH-C, each node sends information about its current location and energy level to the BS. In addition to determining good clusters, the BS needs to ensure that the energy load is evenly distributed among all the nodes. The BS computes the average node energy, and whichever nodes have energy below this average cannot be cluster-heads for the current round. The nodes transmit their data to the $\mathrm{CH}$ node during each frame of data transfer and the $\mathrm{CH}$ aggregates the data and sends the resultant data to the BS. When the $\mathrm{CH}$ node's energy is depleted, the nodes in the cluster lose communication ability with the BS and are essentially dead. Many radio propagation models have been used to predict performance of these protocols using simplified propagation models[12]. They have analyzed and performed simulation using free space model and two ray radio propagation model that fails to reflect actual performance of these 
routing protocols. Therefore, proper modeling of smart grid real environment is needed for proper evaluation of performance of these protocols for WSNs for monitoring and control of the smart grid.

The power grid environments have been modeled using IEEE 802.15.4 compliant WSNs which showed that log normal shadowing path loss model is more accurate channel models to be used in comparing these routing approaches.

The objective of this paper is to simulate and analyze impact of various smart grid environments on performance of four different WSN routing protocols namely two clustering protocols namely the Low Energy Adaptive Clustering Hierarchy (LEACH) and Centralized LEACH (LEACT-C) as well as other two conventional protocols namely Minimum Transmission Energy (MTE) and Static Clustering. The results revealed that smart grid's radio propagation environments have strong impact on the performance of all protocols. The rest of the paper is organized as follows. Section 2 provides description of wireless channel propagation models. Section 3 presents overview of WSN's routing protocols. Section 4 presents simulation of different protocols. Section 5 provides analysis and comparison of the results. Finally, Section 5 provides the conclusion and future work.

\section{Wireless SENSOR Networks Protocols}

WSN's routing protocols are responsible for finding the most efficient path for the messages to travel on its way to a destination. In the following, four protocols namely Minimum Transmission Energy (MTE) and Static Clustering, LEACH and LEACH-C are explained.

\subsection{Minimum TRANSMisSiOn EnERGy (MTE)}

Minimum Transmission Energy (MTE) [13] is a multi-hop routing protocol where sensors communicate directly with other sensors that are within the radio transmission range. Data is passed to each node's next hop neighbor until the data reaches the base station. When a node dies, all of that node's upstream neighbors send their data to the node's next hop neighbor. In this way, new routes do not need to be computed whenever a node dies. Nodes adjust their transmit power to the minimum required to reach their next hop neighbor. This reduces interference with other transmissions and reduces the nodes' energy dissipation. Communication with the next hop neighbor occurs using a Carrier Sense Multiple Access protocol (CSMA) MAC protocol, and when collisions occur, the data are dropped. When a node receives data from one of its upstream neighbors, it forwards the data to its next hop neighbor. This continues until the data reaches the base station. In MTE, each node sends its message to the closest node until it reaches the base station in which sensor nodes serve as routers for other sensor nodes. Therefore, the energy of the sensor nodes that are near to the base station is rapidly consumed.

\subsection{Static Clustering}

In order to enable communication between sensors not within communication range, sensors form clusters using the simulated annealing algorithm as in LEACH-C [14]. Clusters are chosen a priori, each with a cluster head that is selected at the beginning and remain unchanged throughout the lifetime of the network. The static clustering protocol is identical to LEACH except the clusters are chosen a-priori and fixed. Static clustering includes scheduled data communication from the cluster members to the cluster-head and data aggregation at the cluster head. As the cluster head and the cluster remain same for each round, the selected static cluster head die quickly and thus the network lifetime cannot be maximized. 
International Journal of Advanced Smart Sensor Network Systems (IJASSN), Vol 8, No.1/2/3, July 2018

\subsection{LoW EnERgy AdAPTIVe Clustering HierarChy (LEACH)}

In cluster based routing protocols, clustering algorithms are used to divide the network into several clusters based on some stipulated rule. Each cluster is controlled by one or set of node known as Cluster Head $(\mathrm{CH})$. Data is transmitted from member nodes to $\mathrm{CH}$ which fused the collected data and send aggregated data to the central node known as Base Station (BS). LEACH periodically elects $\mathrm{CHs}$ randomly from all the nodes in the networks and rotates this role to balance the energy dissipation of the sensor nodes in the networks. The $\mathrm{CH}$ nodes fuse and aggregate data arriving from nodes that belong to the respective cluster at regular intervals. Network lifetime is divided into discrete and disjoint time intervals called rounds, and every round is composed of two phases, the setup phase and the steady state phase [14]. The setup phase allows clusters to be formulated and $\mathrm{CHs}$ to be elected. In every round, a stochastic algorithm is used by each node to determine whether it will become a $\mathrm{CH}$ or not. If a node becomes a $\mathrm{CH}$ once, it cannot become a $\mathrm{CH}$ again for a given number of rounds.

The selected $\mathrm{CH}$ broadcast an advertisement message to the entire network declaring itself as a new $\mathrm{CH}$. Every node after receiving advertisement message decides its $\mathrm{CH}$ based on received signal strength of advertisement message. After selection of $\mathrm{CH}$, member nodes send message to register with the $\mathrm{CH}$ of their choice. $\mathrm{CH}$ send the time schedule to the registered nodes so that they can send their data using Time Division Multiple Access (TDMA) in MAC layer to allow nodes to turn off their radio component until their allocated time slots. The schedule is broadcasted back to all nodes in the cluster. In the next phase known as steady state phase, sensor nodes can send the observed data to the $\mathrm{CHs}$ on their allocated time slot. $\mathrm{CH}$ send compressed aggregated data to a central station usually know as Base Station (BS). After predetermined time period, new $\mathrm{CHs}$ are elected again randomly. This repetition of electing new $\mathrm{CHs}$ is known as new round so LEACH is based on large number of rounds. Randomization process is used in $\mathrm{LEACH}$ to rotate $\mathrm{CHs}$. During the steady phase, each $\mathrm{CH}$ collects data from all senor nodes in its cluster in their assigned TDMA time slots, aggregates the data, and sends it to the BS along with its cluster head identification.

\subsection{Low Energy Adaptive Clustering Hierarchy- CEnTralized (LeaCh-C)}

In LEACH, the probability of becoming a $\mathrm{CH}$ is based on the assumption that all nodes start with an equal amount of energy, and that all nodes have data to send during each frame. This is not applicable to scenarios where node's energy may vary depending upon their role in the network (.e. whether it is a cluster head or a cluster member). $\mathrm{CH}$ selection plays significant role in developing energy efficient clustering algorithms [10]. Intra cluster communication distance depends upon position of the selected cluster head and intra cluster energy consumption depends upon intra cluster communication distance. Clusters with high intra cluster communication distance consume more energy than other clusters having low intra cluster communication distance. The nodes with more energy are elected more often than the nodes with less energy in order to ensure that all nodes die at approximately the same time. This can be achieved by setting the probability of becoming a $\mathrm{CH}$ as a function of a node's energy level relative to the aggregate energy of the cluster in the network, rather than purely as a function of the number of times, the node has been $\mathrm{CH}$ as in LEACH.

The Low Energy Adaptive Clustering Hierarchy-Centralized (LEACH-C) [15] is adaptable to non-uniform and dynamic energy distribution among the sensor nodes and the changing network configurations. LEACH-C requires that each node transmit information about its location to the base station at the beginning of each round to calculate the average energy of the nodes and whichever nodes have energy below this average will not participate in cluster head selection. The location information is obtained by using a global positioning system receiver that is 
activated at the beginning of each round to get the node's current location. Once clusters are formed and $\mathrm{CHs}$ are selected, BS broadcasts a message that contains the identifications (IDs) of all the CHs. If a node finds it's ID in the message payload, it knows that it has to perform as a $\mathrm{CH}$; otherwise, it determines its allocated TDMA slot and goes to sleep until the transmission time arrives [16]. The steady-state phase of LEACH-C is identical to that of LEACH [17].

\section{SimULATION ENVIRONMENT}

The Network Simulator 2 (NS-2) [18] is used to simulate the four protocols. NS-2 is an open source object oriented discrete event simulator. It has a simulation engine written in $\mathrm{C}++$ to implement protocols and extend the library, with a command and configuration interface using Object Tool Command Language (OTcL). It is a scripting language to create and control the simulation environment itself including the selection of output data. It supports the required features and can be improved and incremented by means of external extensions. Several extensions are added to NS-2 to support simulation of WSNs with various channel propagation and energy dissipation models. IEEE 82.15.4 is not implemented in the default file of NS-2. MAC implementation is developed as recommended. Network topologies are described using the various primitives such as: Nodes, Links, Agents, and Applications. Once the topology has been created, simulations can be run by starting the applications on different nodes at various points in time. WSNs can be observed graphically by Network AniMator (NAM).

The energy, traffic and propagation models are selected to represent smart grid environments. Table 1 gives propagation values for smart grid channel paramters as described in this table.

Extensive simulations to evaluate the performance of the four candidate protocols are simulated for a 100 nodes located in a $200 \mathrm{~m} \times 200 \mathrm{~m}$. Table 2 shows the common simulation parameters and their respective values. The data size is 500 bytes/message plus a header of 25 bytes. The message size to be transmitted is: $X=(500$ bytes +25 bytes $) / 8=4200$ bits.

Table 1: Log Normal Shadowing Channel Parameters

\begin{tabular}{|l|c|c|}
\hline \multicolumn{1}{|c|}{ Propagation Environment } & \multicolumn{1}{c|}{ Path Loss } & Shadowing deviation \\
\hline $\begin{array}{l}\text { 500-kv substation (LOS) } \\
\text { outdoor }\end{array}$ & 2.42 & 3.12 \\
\hline $\begin{array}{l}\text { 500-kv substation (NLOS) } \\
\text { Outdoor }\end{array}$ & 3.51 & 2.95 \\
\hline $\begin{array}{l}\text { Underground network transformer } \\
\text { vault (LOS) }\end{array}$ & 1.45 & 2.54 \\
\hline $\begin{array}{l}\text { Underground network transformer } \\
\text { vault (NLOS) }\end{array}$ & 3.15 & 3.19 \\
\hline $\begin{array}{l}\text { Main power room(LOS) } \\
\text { indoor }\end{array}$ & 1.64 & 3.29 \\
\hline $\begin{array}{l}\text { Main power room(NLOS) } \\
\text { indoor }\end{array}$ & 2.38 & 2.25 \\
\hline $\begin{array}{l}\text { Non smart grid environment } \\
\text { Indoor environment }\end{array}$ & 1.4 & 4 \\
\hline
\end{tabular}

\section{Performance Metrics}

The most important metrics for evaluating WSNs are number of data received at base station, energy consumption, network lifetime. Statistics are collected at periodic time intervals to evaluate the effectiveness of the different candidate protocols. 


\section{(a) Data Received At Base Station}

It is needed to know that how much data is received at the base station from the sensor networks. If the amount of data received is enough as it is expected then the network will perform well.

\section{(b) Average Energy Consumption}

The average energy consumption is calculated across the entire topology. It measures the average difference between the initial level of energy and the final level of energy that is left in each node. If $E_{i}$ is the initial energy level of a node, $E_{f}$ is the final energy level of a node and $N$ is number of nodes in the simulation, then the average energy consumption is

$$
E_{a}=\frac{\sum_{k=1}^{n}\left(E_{i k}-E_{f k}\right)}{N}
$$

This metric is important because it is proportional to the network's lifetime. The lower the energy consumption the longer is the network's lifetime.

\section{(c) Total Number of Nodes Alive}

This metric indicates the overall lifetime of the network. Performance of a network depends on the lifetime of each node, if the lifetime of the nodes increase then the network performs well and sensors transmit more data to the base station. A lower value of the energy consumption metric and a higher number of nodes alive at any given time indicates a more efficient protocol.

Table 2: NS-2 Simulation Parameters

\begin{tabular}{|c|c|}
\hline Simulator & NS-2.34 \\
\hline Examined Protocols & MTE, Static Clustering, LEACH, and LEACH-C \\
\hline Simulation Duration & 1000 seconds \\
\hline Simulation Area & $200 \mathrm{~m} \times 200 \mathrm{~m}$ \\
\hline Number of Nodes & 100 \\
\hline Location of BS & 50,175 \\
\hline Initial Energy & 2 Joules \\
\hline Number of cluster heads & $5 \%$ \\
\hline $\mathrm{E}_{\text {elec }}$ & 50 nano Joule / bit \\
\hline$\varepsilon_{\mathrm{fs}}$ & 10 pico Joule/bit $/ \mathrm{m}^{2}$ \\
\hline$\varepsilon_{\mathrm{amp}}$ & 0.0015 pico Joule/ bit $/ \mathrm{m}^{4}$ \\
\hline Crossover Distance & $87 \mathrm{~m}$ \\
\hline Radio propagation speed & $3 \times 10^{8} \mathrm{~m} / \mathrm{s}$ \\
\hline Radio Speed & $1 \mathrm{Mbps}$ \\
\hline Antenna gain $\left(\mathbf{G}_{\mathbf{t}}, \mathbf{G}_{\mathbf{r}}\right)$ & 1 \\
\hline Antenna height $\left(\mathbf{h}_{\mathbf{t}}, \mathbf{h}_{\mathbf{r}}\right)$ & $1.5 \mathrm{~m}$ \\
\hline System Loss Factor L & 1 \\
\hline Signal Wavelength & $0.328 \mathrm{~m}$ \\
\hline Data payload & 250 Bytes \\
\hline Radio Bit Rate & $1 \mathrm{Mbps}$ \\
\hline Processing Delay & 50 micro seconds \\
\hline
\end{tabular}

\section{Simulation RESUlts AND ANAlysiS}

The four protocols were simulated first in ideal two ray ground propagation and then compared with shadowing propagation model. Although the two-ray propagation model has been widely used WSN's simulation, this model is inappropriate as it is based on simplified assumptions that 
neglect the effect of fading, which represents the actual environments. The two ray ground has delivered more data to base station, consumed less energy, and has longer lifetime. The results revealed that the realistic and representative shadowing propagation model under harsh smart grid environments have considerable impact on reliability and network lifetime of the four protocols. Consequently, the performance deteriorated very quickly when shadowing model was taken into account. The main reasons for this deterioration resulted from the large variation of the received signal strength. Hence packets are not received successfully at BS due to the poor signal quality, which causes problems to the normal operations of various protocols. The four protocols performed quite differently and this gives a hint to the fact that simulation results for WSNs have to be interpreted with a lot of care in order to conclude accurate results especially when reliability and network lifetime are considered. Due to space limitations, results for two ray ground is not shown here but it is available upon request.

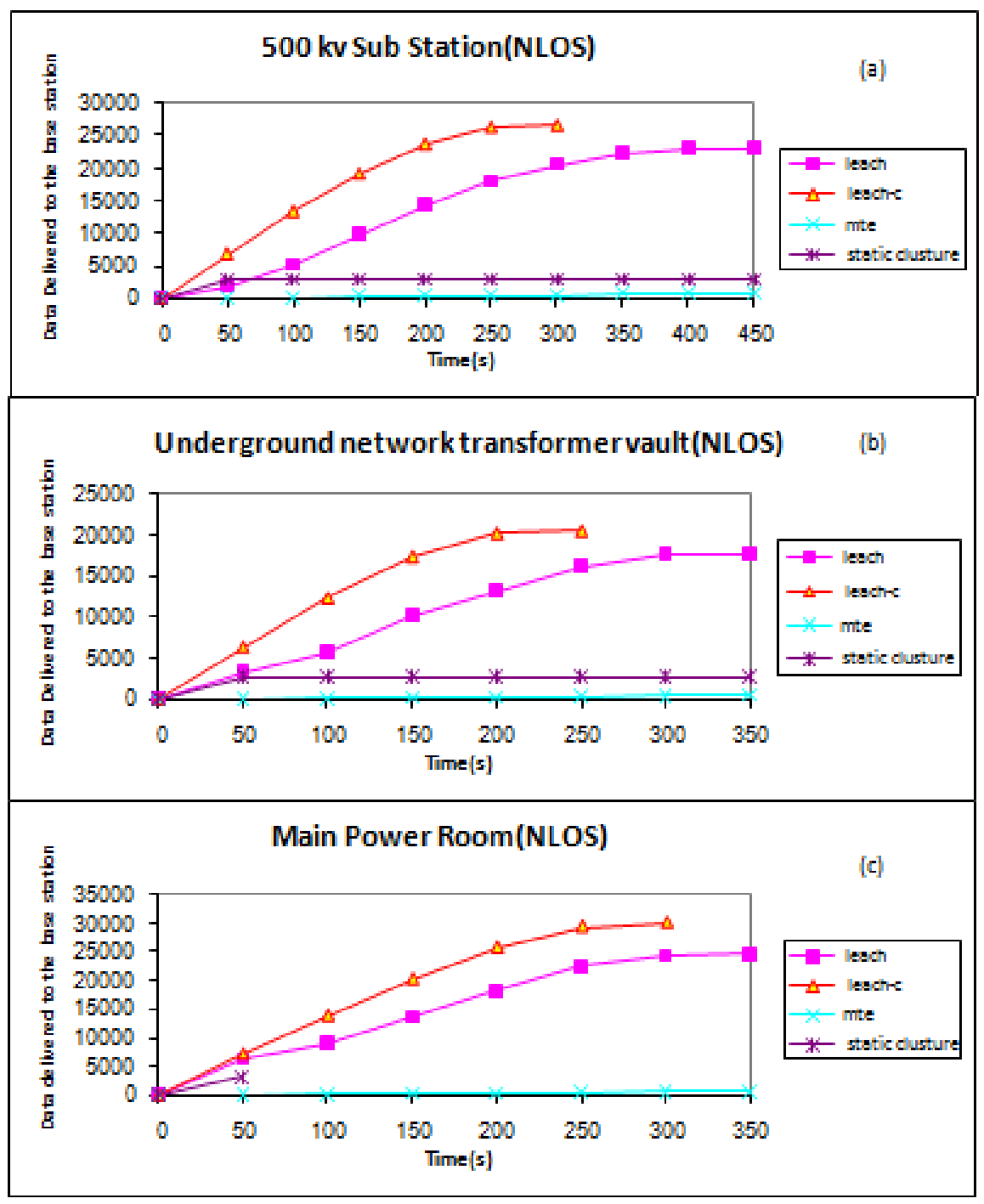

Figure 1. Total Number of Data Delivered to Base Station vs. Time in (a) 500 kV Sub-Station-NLOS, (b) Main Power Room-NLOS, (c) Underground Transformer Vault-NLOS 
The aim of this paper is to simulate different WSNs protocols and analyze their performance using the realistic and representative shadowing propagation model under harsh smart grid environments. Due to limited space, only three environments are presented here corresponding to a $500 \mathrm{kV}$ substation NLOS, an underground transformer vault NLOS, and a main power room NLOS environments. Results for the other four environments are available upon request. All over the simulation, the seven different environments have considerable impact on performance metrics and the different protocols exhibit nearly the same behavior over time in different environments.

In Figure 1, LEACH-C delivers the highest amount of data to base station and considerably outperforms LEACH in terms of the amount of data sent to the base station during network lifetime as depicted in Figure 1. This is because the base station has global knowledge of the location and energy of all the nodes in the network. Simulation time of LEACH-C ends earlier. Both protocols outperform MTE and static clustering because both rotate selection of CHs between nodes and adapt the corresponding clusters based on the nodes that are selected to be cluster heads at a given time. In static clustering, nodes are organized into clusters initially by the BS using the same method as in LEACH-C to ensure that good clusters are formed. These clusters and cluster heads remain fixed throughout the lifetime of the network. Nodes transmit their data to the cluster head node during each frame of data transfer and the cluster head aggregates the data and sends the resultant data to the BS. When the cluster head node's energy is depleted, the nodes in the cluster lose communication ability with the BS.As soon as the cluster head node dies, all nodes from that cluster effectively die since there is no way to deliver their data to the base station as shown in Figure 2.

In Figure 2, the energy consumption for MTE routing increases with simulation time. Each node runs a start-up routine to determine its next-hop neighbor, defined to be the closest node that is in the direction of the BS. Data packets are passed along via next hop neighbors until they reach the BS. As there is no central control in MTE routing, it is difficult to set up fixed MAC protocols (e.g., TDMA), so each node uses CSMA to listen to the channel before transmitting data. If the channel is busy, the node backs off; otherwise, the node transmits its data to the next-hop node. As nodes run out of energy, the routes are recomputed to ensure connectivity with the BS. As time increases, more nodes die and this reduce amount of energy consumed with respect to other protocols. Figure 2 also shows that LEACH consumes less amount of energy than LEACH-C. LEACH is completely distributed which requires no control information from the base station, and the nodes do not require knowledge of the global network in order for LEACH to operate. Distributing the energy among the nodes in the network is effective in reducing energy consumption from a global perspective and enhancing network lifetime. 
International Journal of Advanced Smart Sensor Network Systems (IJASSN), Vol 8, No.1/2/3, July 2018

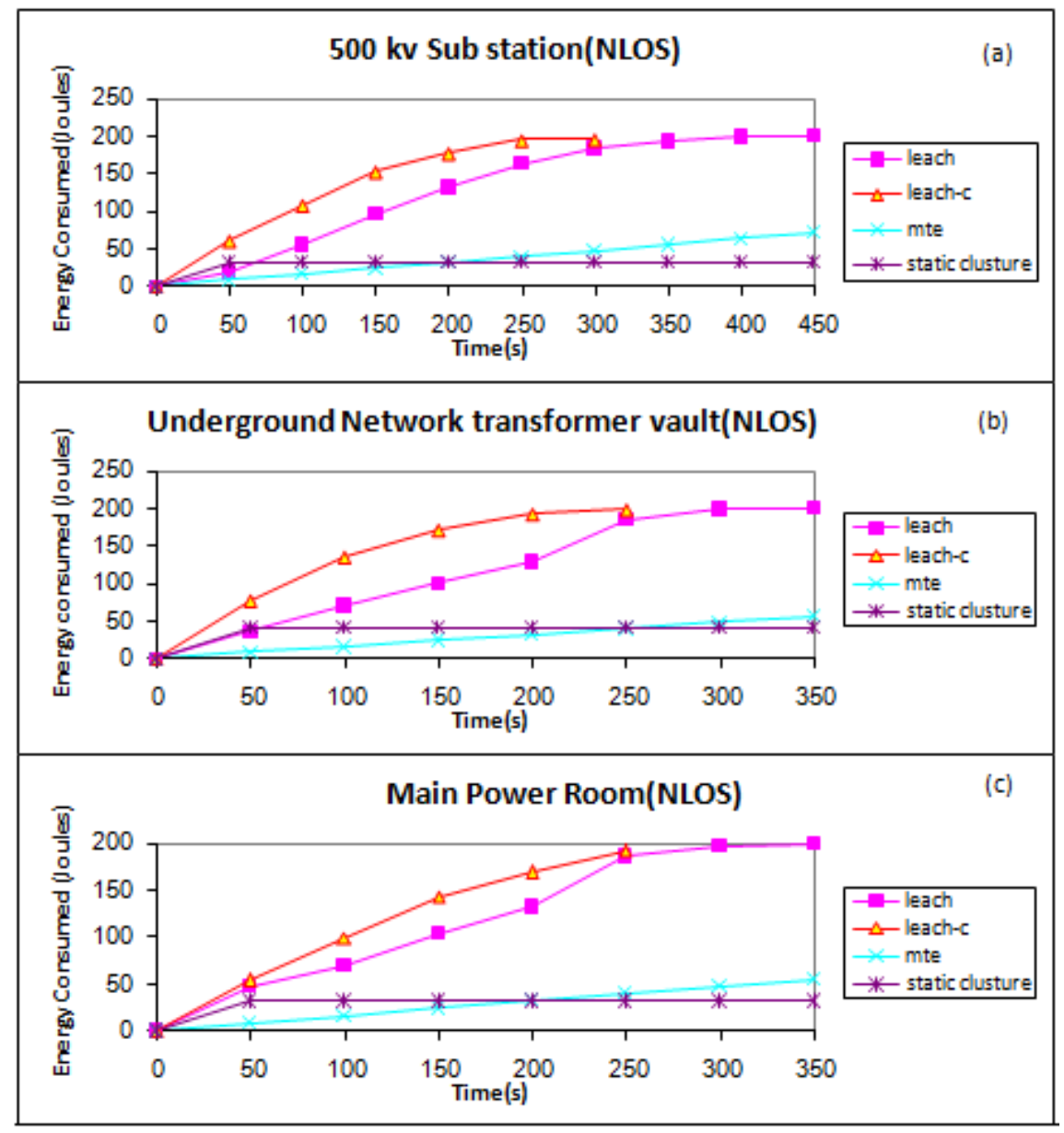

Figure 2: Average Energy Consumption Vs. Simulation Time in (a) 500 kV Sub-Station-NLOS, (b) Main Power Room-NLOS, (c) Underground Transformer Vault-NLOS

Figure 3 shows the number of nodes that remains alive using LEACH is significantly larger than that of LEACH-C. Lifetime of LEACH is longer than LEACH-C as LEACH rotates the cluster head nodes and the associated clusters, nodes die more slowly which indicate that the protocol can balance the nodes' energy consumption. In addition to reducing energy consumption in last figure, LEACH successfully distributes energy usage among the nodes in the network such that the nodes die randomly and at essentially the same rate. However, the main problem with the LEACH protocol lies in the random selection of CHs. There exists a probability that the $\mathrm{CH}$ formation is unbalanced and may remain in one part of the network, making some part of the network unreachable. In MTE, all node pass own data to nodes closest to BS and that nodes die quickly due to higher energy consumption. In static clustering, as soon as the cluster head node dies, all nodes from that cluster effectively die since there is no way to deliver their data to the base station although member nodes have enough energy. The nodes in static clustering are completely depleted in the first 50 seconds which indicate that static clustering performs poorly because more energy is drained from preassigned fixed cluster heads due to message transmission over long distances compared to other nodes in the cluster based protocols. Therefore, cluster head node's energy is drained quickly, ending the lifetime of all other nodes belonging to those clusters as they lose communication with base station. 
International Journal of Advanced Smart Sensor Network Systems (IJASSN), Vol 8, No.1/2/3, July 2018

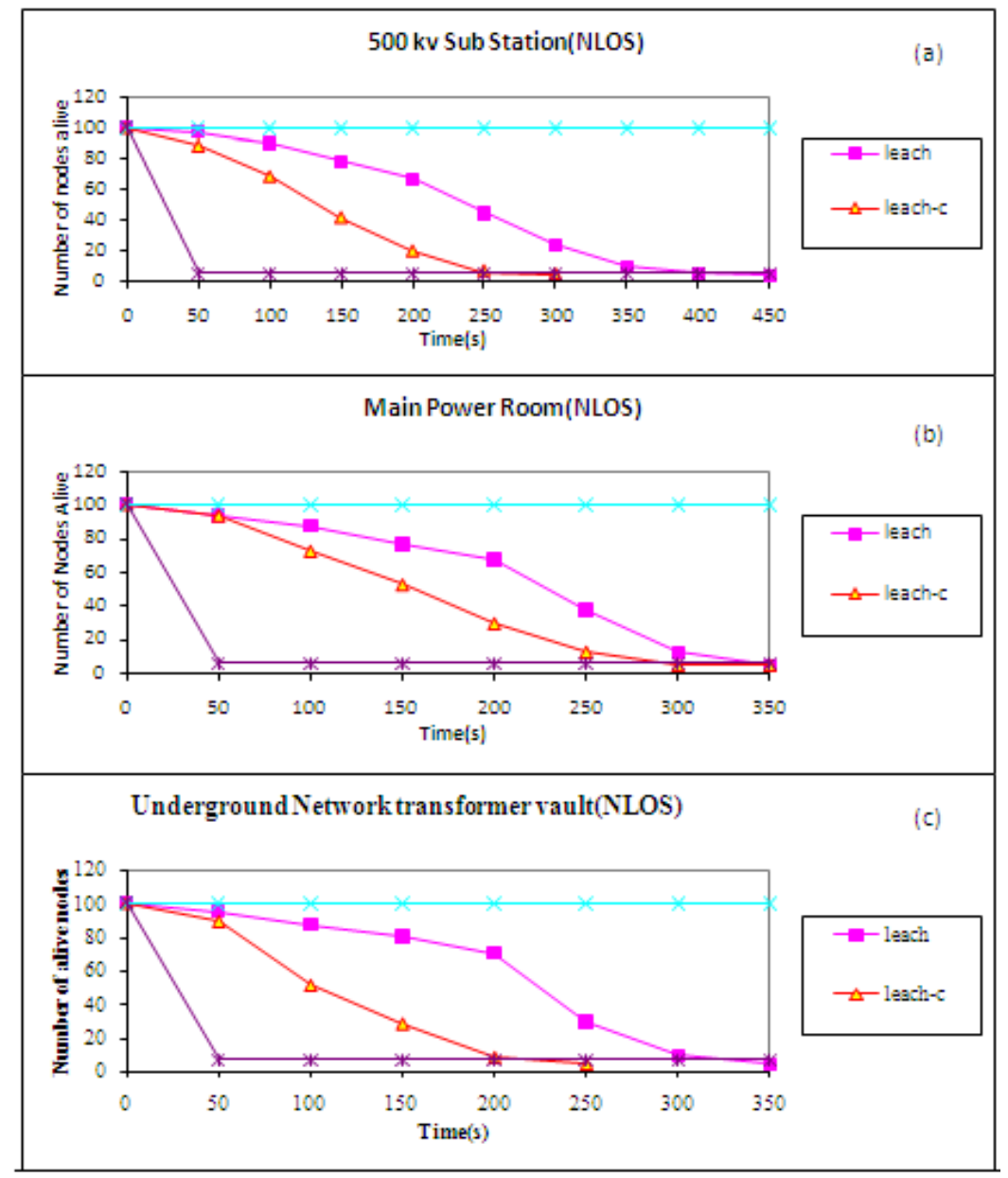

Figure 3: Total Number of Alive Nodes Vs. The Simulation Time in (a) $500 \mathrm{kV}$ Sub-Station-NLOS, (b) Main Power Room-NLOS, (c) Underground Transformer Vault-NLOS

Figure 4 shows that static clustering and MTE deliver lowest amount of data to base station while LEACH and LEACH-C deliver the highest amount of data per unit energy, achieving energy efficiency. LEACH-C deliver more data per unit energy than LEACH because the BS has global knowledge of the location and energy of all the nodes in the network, so it can produce better clusters that require less energy for data transmission. Static clustering performs poorly because all $\mathrm{CH}$ nodes die quickly, ending the lifetime of all nodes belonging to those clusters. It is shown that LEACH is almost as efficient as LEACH-C. In LEACH-C it starts with the lower energy consumption but due to the transmission overhead it requires high energy consumptions. LEACH is found to be most energy efficient among the four protocols. LEACH is completely distributed which requires no control information from the base station, and the nodes do not require knowledge of the global network in order for LEACH to operate.

Figure 5 shows the total number of nodes that remain alive per amount of data received at the BS. LEACH can deliver more effective data than LEACH-C for the same number of node deaths. MTE requires the highest amount of energy to send data to the BS due to collisions and lack of data aggregation. Also MTE does not have any centralized control over when nodes transmit and receive packets, collisions increase the amount of energy required to send each successful message, causing more node deaths for the same amount of data delivery to end network lifetime early. Furthermore, each message in MTE must traverse multiple hops to reach

$\mathrm{BS}$, whereas each 
International Journal of Advanced Smart Sensor Network Systems (IJASSN), Vol 8, No.1/2/3, July 2018
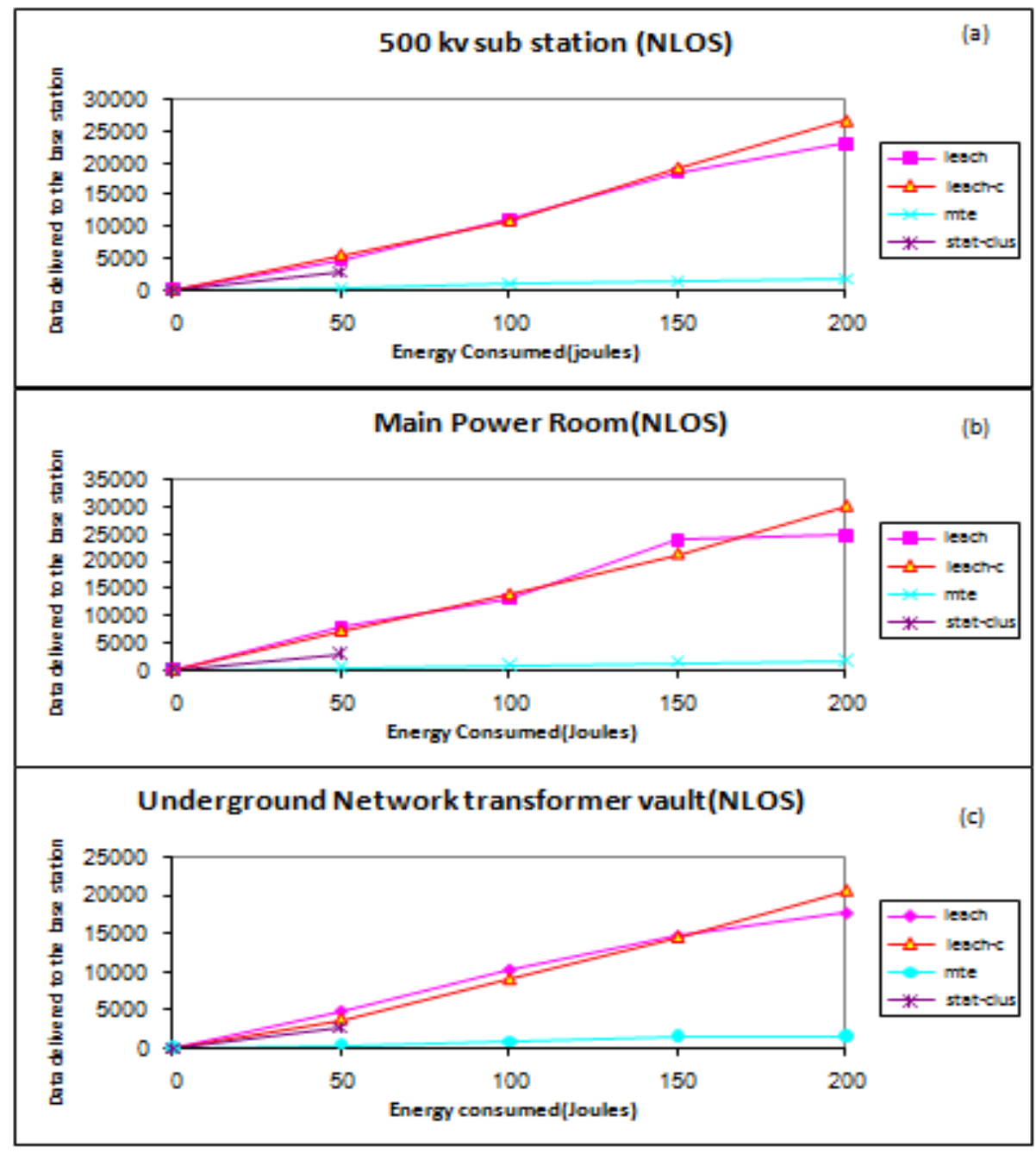

Figure 4: Average Energy Consumption Vs. Simulation Time in (a) 500 kV Sub-Station-NLOS, (b) Main Power Room-NLOS, (c) Underground Transformer Vault-NLOS

message in LEACH need only traverse one hop due to data aggregation at the cluster head. The static clustering performs poorly and the cluster head nodes die quickly, ending the lifetime of all nodes belonging to those clusters. The static clustering drains the nodes quickly as nodes die early and loose communications with BS. As the cluster head and the cluster remain same for each round, the selected static cluster heads die quickly and thus the network lifetime is quickly degraded. The figures clearly show the large advantage of using LEACH and LEACH-C versus conventional protocols in terms of network lifetime for a given amount of received data at the base station and LEACH outperforms LEACH-C in prolonging network lifetime. 


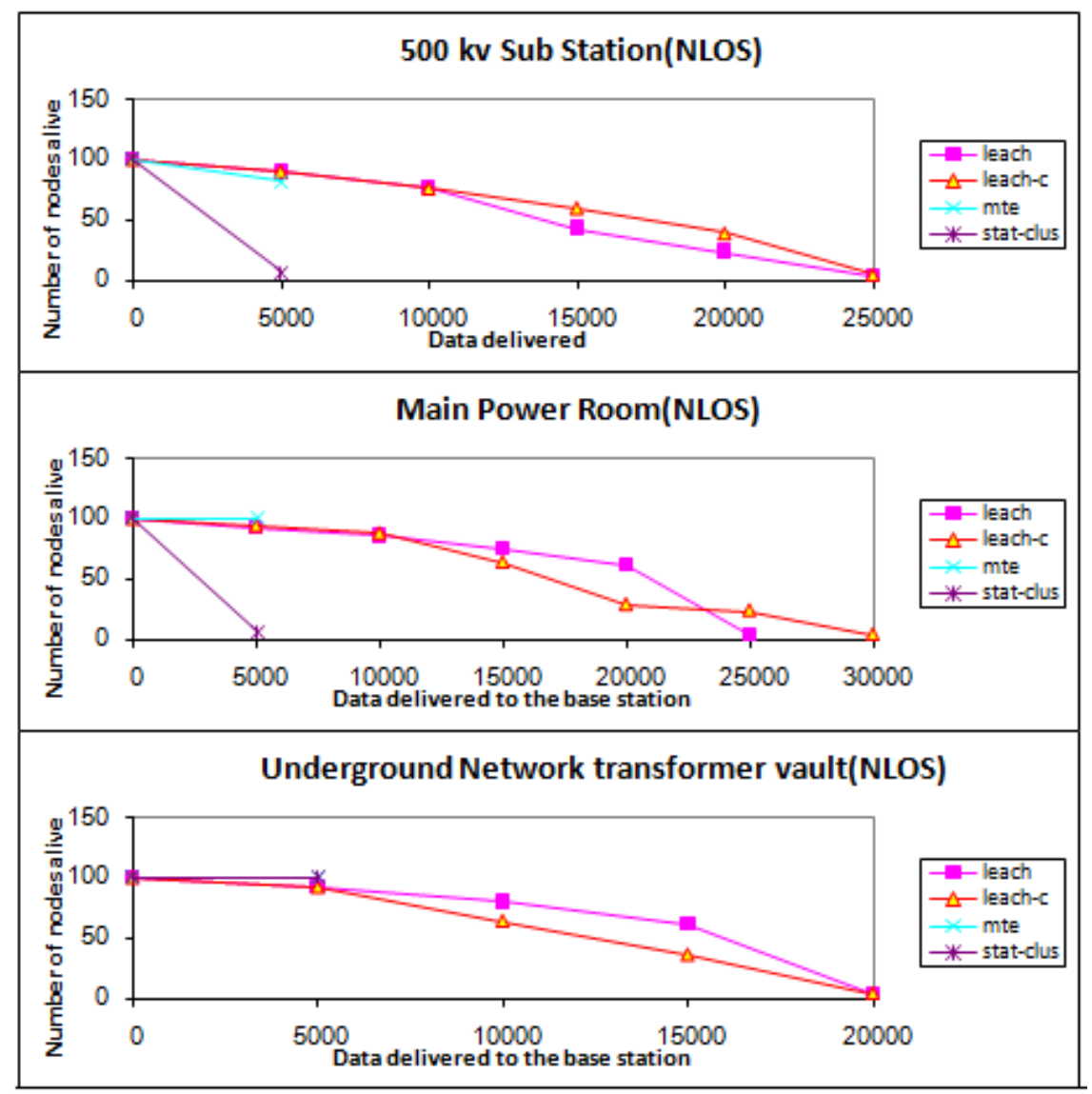

Figure 5: Number of alive nodes vs. number of data received at the base station in (a) $500 \mathrm{kV}$ Sub-StationNLOS, (b) Main Power Room-NLOS, (c) Underground Transformer Vault-NLOS

\section{CONCLUSION AND Future WORK}

Latest advances in computing and networking have enabled WSNs to realize ambient intelligence, which is a vision through which electric power grid becomes smart. The main challenge in the design of routing protocols for WSNs is to ensure effective consumption of energy with ultimate objective to extend lifetime of network for as long as possible. The realization of smart grid depends on communication facilities of WSNs in harsh and complex environments of electric power grid that have been modelled using shadowing model. To achieve reliable wireless communications within WSNs, it is essential to investigate performance of routing protocols in different smart grid environments. Four WSNs protocols namely MTE, Static Clustering, LEACH, and LEACH-C are simulated on NS- 2. The performance is evaluated using number of data signals received at base station, energy consumption, and network lifetime as performance metrics. The paper analyzed the results and finally reaches to a conclusion about the routing protocol that can be efficient for monitoring and control applications in the smart grid.

The results have clearly showed that the smart grid environments have directly affected performance of various protocols and energy efficient clustering approaches are more effective in prolonging the network lifetime compared to conventional protocols. It is clear that in MTE routing is not suitable for smart grid applications as the nodes closest to the base station will be used to route a large number of data messages to the base station. Thus, these nodes will die out quickly, causing the energy required to get the remaining data to the base station to increase and 
more nodes to die. This will create a cascading effect that will shorten network lifetime. In addition, as nodes close to the base station die that area of the environment is no longer being monitored. In addition, static clustering is not suitable, where nodes are organized into clusters that communicate with a local base station, and these local base stations transmit the data to the global base station, where it is accessed by the end user. However, the local base station is assumed to be ahigh-energy node; if the base station is an energy constrained node, it would die quickly, as it is being heavily utilized.

LEACH-C achieved considerable improvement in number of data derived to base station because it select cluster heads based on remaining energy and their geographical position. However, it consume more energy and shorten the network lifetime. On the other hand, LEACH is completely distributed which requires no control information from the base station, and the nodes do not require knowledge of the global network in order for LEACH to operate. Distributing the energy among the nodes in the network is effective in reducing energy consumption from a global perspective and enhancing network lifetime. In all simulation, LEACH provided considerable energy savings and prolonged network lifetime over other protocols. As the protocol is fully distributed so there is no intervention from Base station in cluster formation and cluster head selection. Global location knowledge of sensor nodes is not required. These features makes LEACH a promising option for various smart grid applications.

Some shortcomings however are uneven distribution of $\mathrm{CH}$ and unbalance energy utilization in the whole network. Due to random selection of $\mathrm{CH}$ it could not guaranteed that Cluster heads are evenly distributed in the network. LEACH assumes that energy of all nodes is same and remains fixed with time. It also assumes that energy consumption across the network is identical as the cluster head selection is rotated among the nodes. These assumptions make $\mathrm{CH}$ selection unrelated to the residual energy of a node and nodes with little energy are as likely to become cluster heads as the nodes with abundance of energy. The energy deficient nodes die fast and gradually render the network useless even though there might be numerous nodes still having enough energy to be functional. Moreover, even though the nodes are equipped with the same energy at the beginning, the networks cannot evolve equably for each node in expending energy, due to the radio communication characteristics, random events such as short-term link failures or morphological characteristics of the fields. Therefore, WSNs are possibly heterogeneous networks and protocols should meet the need of the characteristic of heterogeneous wireless sensor networks. Due to these drawbacks, future work will be carried out to solve these problems and to propose an adaptive and reliable cluster based protocol to enhance energy consumption and prolonging networks lifetime in smart grid environments.

\section{REFERENCES}

[1] E. Fadel, V.C. Gungor, Laila Nassef, Nadine Akkari, M.G. Abbas Malik, Suleiman Almasri and Ian F. Akyildiz, "A Survey on Wireless Sensor Networks For Smart Grid," Computer Communications, Vol. 71, pp. 22-33, November 2015, https://doi.org/10.1016/j.comcom.2015.09.006.

[2] M. Hammoudeh and R. Newman, "Adaptive routing in wireless sensor networks: QoS optimisation for enhanced application performance," Information Fusion, Vol. 22, pp. 03-15, March 2015, https://doi.org/10.1016/j.inffus.2013.02.005.

[3] M. R. Mundada, S. Kiran, S. Khobanna, R. N. Varsha and S. A. George, "A STUDY ON ENERGY EFFICIENTA STUDY ON ENERGY EFFICIENT SENSOR NETWORKS," International Journal of Distributed and Parallel Systems (IJDPS), Vol.3, No.3, pp. 311-330, May 2012, DOI : 10.5121/ijdps.2012.3326.

[4] F. Farazandeh, R. Abrishambaf and S. Uysal, "A Hybrid Energy-Efficient routing protocol for Wireless Sensor Networks," in 11th IEEE International Conference on Industrial Informatics (INDIN), Bochum, Germany, 2013, DOI: 10.1109/INDIN.2013.6622851.

[5] P. Yuvaraj and Komanapalli Venkata Lakshmi Narayana, "EESCA: Energy efficient structured 
International Journal of Advanced Smart Sensor Network Systems (IJASSN), Vol 8, No.1/2/3, July 2018

clustering algorithm for wireless sensor networks," in International Conference on Computing, Analytics and Security Trends (CAST), Pune, India, 19-21 Dec. 2016, DOI: 10.1109/CAST.2016.7915024.

[6] S. C. Sharma and S. P. Singh, "A Survey on Cluster Based Routing Protocols in Wireless Sensor Networks," Procedia Computer Science, vol. 45, pp. 687-695, 2015, https://doi.org/10.1016/j.procs.2015.03.133.

[7] Z. A. Sadouq, M. El Mabrouk and M. Essaaidi, "Conserving Energy in WSN Through Clustering and Power Control," in Third IEEE International Colloquium in Information Science and Technology (CIST), Tetouan, Morocco, 20-22 Oct. 2014, DOI: 10.1109/CIST.2014.7016654.

[8] N. Ramluckun and V. Bassoo, "Energy-efficient chain-cluster based intelligent routing technique for Wireless Sensor Networks," Applied Computing and Informatics, 07 March 2018, https://doi.org/10.1016/j.aci.2018.02.004.

[9] Pooja and S. Singh, "Improved O-LEACH protocol: A clustering based approach in wireless microsensor network," in 10th International Conference on Intelligent Systems and Control (ISCO), Coimbatore, India, 7-8 Jan. 2016, DOI: 10.1109/ISCO.2016.7727015.

[10] W. B. Heinzelman, "A Component-Based Cross-Layer Framework for Software Defined Wireless Networks," in 8th IFIP International Conference on New Technologies, Mobility and Security (NTMS), Larnaca, Cyprus, 21-23 Nov. 2016, DOI: 10.1109/NTMS.2016.7792426.

[11] M. Tripathi, M. S. Gaur, V. Laxmi and R. B. Battula, "Energy efficient LEACH-C protocol for Wireless Sensor Network," in CIIT 2013. Third International Conference on Computational Intelligence and Information Technology, Mumbai, India, 18-19 Oct. 2013, DOI: 10.1049/cp.2013.2620.

[12] L. Nassef, "On the Effects of Fading and Mobility in On Demand Routing Protocols," Egyptian Informatics Journal, Vol.11, pp. 67-74, 2010, http://dx.doi.org/10.1016/j.eij.2010.10.003.

[13] B. Pati, J. L. Sarkar and C. R. Panigrahi, "ECS: An Efficient Approach to Select Cluster Head in Wireless Sensor Networks," Arabian Journal for Science and Engineering, VoL. 42, Issue: 2, p. 669676, February 2017, https://doi.org/10.1007/s13369-016-2304-2.

[14] R. D. Gawade and S. L. Nalbalwar, "A Centralized Energy Efficient Distance Based Routing Protocol for Wireless Sensor Networks," Journal of Sensors, Vol. 2016, 2016 , http://dx.doi.org/10.1155/2016/8313986.

[15] I. Sharma, R. Singh and M. Khurana, "Comparative study of LEACH, LEACH-C and PEGASIS routing protocols for wireless sensor network," in International Conference on Advances in Computer Engineering and Applications (ICACEA), Ghaziabad, India, 19-20 March 2015, DOI: 10.1109/ICACEA.2015.7164821.

[16] V. Geetha, P. V. Kallapur and S. Tellajeera, "Clustering in Wireless Sensor Networks: Performance Comparison of LEACH \& LEACH-C Protocols Using NS2," Procedia Technology, Vol. 4, pp. 163170, 2012, https://doi.org/10.1016/j.protcy.2012.05.024.

[17] W. Xinhua and W. Sheng, "Performance comparison of LEACH and LEACH-C protocols by NS2," in Ninth International Symposium on Distributed Computing and Applications to Business Engineering and Science (DCABES), Hong Kong, China, 10-12 Aug. 2010.

[18] "http://www.isi.edu/nsnam/ns/," The network simulator ns-2. [Online]. 